with demy quarto, and the printed page is too large to allow of the paper being cut down to demy or even medium octavo size. The Atti of the Lincei Academy may be cited as an important example. Fortunately, however, such exceptions are comparatively few in number, and they include none of the main English journals in which original papers are published on mathematics or physics.

It is my hope that by again directing attention to this matter further uniformity may be secured in the sizes of proceedings and transactions by the gradual elimination of inconvenient sizes, and by the avoidance of further divergences. The size of the new number of London Mathematical Proceedings is peculiarly unfortunate, as it is not uniform even with those outstanding foreign journals which do not conform to the recommendations of the committee.

G. H. BryaN.

\section{Expansion Curves.}

Mr. Stodnart's method of finding points on the curve $p z^{\prime \prime}=$ constant, to which Prof. Perry directed attention on October 8 (p. 548), is interesting, but it does not give a great number of convenient points on the curve. If the points $\mathrm{A}, \mathrm{E}, \ldots$ are called $\left(v_{1}, p_{1}\right),\left(v_{2}, p_{2}\right), \ldots$ the values of $v$ and $p$ form two series of quantities in continued proportion, i.e. such that $v_{1} / v_{2}=v_{2} / v_{3}=\ldots$, and $p_{1} / p_{2}=p_{2} / p_{3}=\ldots$

A modification of the method, bringing out more clearly its essential simplicity, and, moreover, far easier in practice, would be to calculate the positions of two points $A, E$ instead of finding $A$ and the specially related angles $\alpha, \beta$.

It will then be seen from the diagram, by drawing the lines noedful to find a third point (say for definiteness in

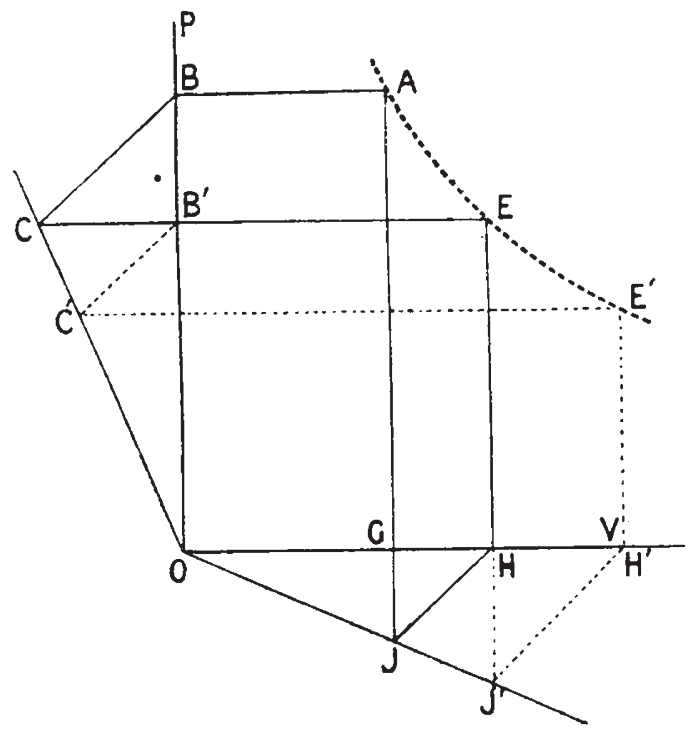

the direction of increasing $v$ and decreasing $p$ ), that the method advocated is only that of fonding the above two series of continued proportionals, and that any angles would serve the purpose, all that is necessary being that all the construction lines like $\mathrm{JH}$ must be parallel to each other, and similarly all the lines like $B C$ parallel to each other. But no modification of the method will give more than the points I have indicated.

By drawing the tangents at the points so found, the accurate construction of the curve would evidently be facilitated. This can be neatly done by taking care in the choice of the first two points; for in these curves the gradient is $-p \div v / n$, so that the tangent at the point $(v, p)$ cuts the axis of $x$ at the point the abscissa of which is $v(1+1 / n)$. Hence if we choose the first two points $\left(v_{1}, p_{1}\right)$ and $\left(v_{2}, p_{2}\right)$ so that $v_{2}=v_{1}(1+1 / n)$, the tangent at the first point will pass through the foot of the second ordinate, and similarly the tangent at the second point will pass through the foot of the third ordinate, and so on.

No. 1773 . VOL. 68]
Or, if we take $v_{2}=v_{1} \vee(1+1 / n)$, so that $v_{3}=v_{1}(1+1 / n)$, the first tangent will pass through the foot of the third ordinate, and so on.

This happens to be approximately the case in Prof. Perry's diagram, which for convenience has been reproduced here with a set of additional construction lines.

Coopers Hill.

Alfred LOdGe.

\section{Rocket Lightning.}

A peculiar species of lightning, bearing a strong resemblance to ascending rockets, was witnessed on the evening of July 22 by two of the professors in Sibpur Engineering College, Howrah, near Calcutta, one of whom wrote me the following careful account in a letter dated the next day. I wrote back suggesting local inquiry in the direction in which the phenomenon appeared, and sending some extracts from Hann's "Lehrbuch der Meteorologie" bearing on the subject. The reply, dated September I, shows that the suggested inquiry is impracticable.

I I Leopold Road, Ealing, W.

J. D. EveretT.

We saw some strange lightning yesterday evening at about 9 p.m. It was a clear, moonless night, with just a bank of cloud very low in the S.S.W., with a well-marked edge, height say from horizon (flat) to $5^{\circ}$ up. There was a misty cloud above this. These clouds we could only see properly when the flashes came. Stars were visible at about $10^{\circ}$ above the horizon at this point, and the sky was quite clear all over elsewhere. Now and then flashes showed from behind the lower cloud (the flashes themselves were mostly hidden, and thunder was not audible). The flashes were not so frequent as usual, say one per minute or so. Generally here they are almost incessant during thunderstorms.

At intervals of three minutes or so, immediately after a flash-which, as common here, was mostly multiple, lasting a second or so altogether-a luminous trail shot straight up to $15^{\circ}$ or so, about as fast as, or rather faster than, a rocket, and of very similar appearance, but with minute waves, like ribbon lightning. It was hardly as bright as most lightning. $S$. and $I$ saw it repeated seven times, and Prof. Brühl (physics) three or four times after we directed his attention to it. He was equally surprised at the novelty, and he has been out here some eighteen years. One of the trails turned off, as shown; the others were about vertical as seen from here. Each grew up steadily from below, and then disappeared at once. The upper end was definite, and did not branch or spread.

In each case it followed immediately on a vivid flash or set of flashes. It was certainly not fireworks of any kind. It terminated in apparently clear sky. Its appearance as a uniformly and very bright ribbon was different from any freworks. It was somewhat yellowish, not purple as lightning often is. It was much too far off for fireworks to be so high and bright. No thunder was audible. July 23.

W. H. Evfrett.

Thanks for trouble of making extracts from Hann re lightning, which, as you say, describe phenomena different from what we saw.

Peake is in charge of the Meteorological Office for India and did not hear about it, nor did $I$ see anything in the Calcutta newspapers.

There would be practically no Europeans or any competent observers nearer the lightning than we were; as it must have occurred at a spot above the Sunderabunds, a wilderness of waterways and jungle. And there are probably not a score of men in all Bengal who would take serious interest in such lightning if they did happen to see it. I was lucky to have Brühl as a witness, he being an old resident, and one who keeps his eyes open.

It was not like a string of fireballs, for it was of uniform width. But it had, as Hann says of globular lightning, doubtless some connection with the breaking down of the air by the volleys of discharged.

September I. 